- Most oral malodour is related to diet, habits or inadequate oral hygiene.

- However cancer and some systemic and psychogenic diseases may present with malodour

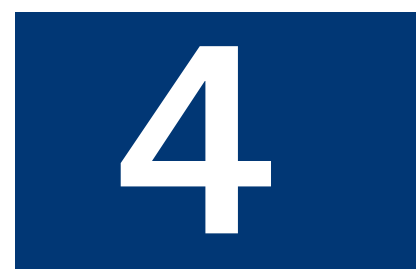

\title{
Oral Medicine - Update for the dental practitioner Oral malodour
}

\author{
C. Scully ${ }^{1}$ and D. H. Felix ${ }^{2}$
}

This series provides an overview of current thinking in the more relevant areas of oral medicine for primary care practitioners, written by the authors while they were holding the Presidencies of the European Association for Oral Medicine and the British Society for Oral Medicine, respectively. A book containing additional material will be published. The series gives the detail necessary to assist the primary dental clinical team caring for patients with oral complaints that may be seen in general dental practice. Space precludes inclusion of illustrations of uncommon or rare disorders, or discussion of disorders affecting the hard tissues. Approaching the subject mainly by the symptomatic approach - as it largely relates to the presenting complaint - was considered to be a more helpful approach for GDPs rather than taking a diagnostic category approach. The clinical aspects of the relevant disorders are discussed, including a brief overview of the aetiology, detail on the clinical features and how the diagnosis is made. Guidance on management and when to refer is also provided, along with relevant websites which offer further detail.

\section{ORAL MEDICINE \\ 1. Aphthous and other common ulcers \\ 2. Mouth ulcers of more serious connotation \\ 3. Dry mouth and disorders of salivation \\ 4. Oral malodour \\ 5. Oral white patches \\ 6. Oral red and hyperpigmented patches \\ 7. Orofacial sensation and movement \\ 8. Orofacial swellings and lumps \\ 9. Oral cancer \\ 10. Orofacial pain \\ "Professor, Consultant, Dean, Eastman Dental Institute for Oral Health Care Sciences, 256 Gray's Inn Road, UCL, University of London, London WC1X 8LD; ${ }^{2}$ Consultant, Senior Lecturer, Glasgow Dental Hospital and School, 378 Sauchiehall Street, Glasgow G2 3JZ | Associate Dean for Postgraduate Dental Education, NHS Education for Scotland, 2nd Floor, Hanover Buildings, 66 Rose Street, Edinburgh EH2 2NN \\ ${ }^{*}$ Correspondence to: Professor Crispian Scully CBE \\ Email:c.scully@eastman.ucl.ac.uk}

\section{Refereed Paper}

( ) British Dental Journal 2005; 199 498-500

\section{ORAL MALODOUR}

Oral malodour, or halitosis, is a common complaint in adults, though few mention it. Malodour can have a range of causes (Table 1). With oral malodour from any cause, the patient may also complain of a bad taste.

\begin{tabular}{l} 
Table 1 Main causes of oral malodour \\
\hline Oral sepsis \\
Dry mouth \\
Starvation \\
Some foods \\
Habits: smoking, alcohol and some drugs \\
Systemic disease \\
$\quad$ Diabetic ketosis \\
$\quad$ Gastrointestinal disease \\
Hepatic failure \\
Renal failure \\
Respiratory disease \\
Trimethylaminuria \\
Psychogenic factors
\end{tabular}

\section{Common causes of oral malodour}

Oral malodour is common on awakening (morning breath) and then often has no special significance - usually being a consequence of low salivary flow and lack of oral cleansing during sleep as well as mouthbreathing.

This rarely has any special significance, and can be readily rectified by eating, tongue brushing, and rinsing the mouth with fresh water. Hydrogen peroxide rinses may also help abolish this odour.
Oral malodour at other times is often the consequence of eating various foods such as garlic, onion or spices, foods such as cabbage, Brussel sprouts, cauliflower and radish, or of habits such as smoking, or drinking alcohol. Durian is a tropical fruit which is particularly malodourous.

The cause of malodour in such cases is usually obvious and avoidance of the offending substance is the best prevention.

\section{Less common causes of oral malodour}

Oral infections can be responsible for oral malodour. The micro-organisms implicated in oral malodour are predominantly Gram-negative anaerobes, and include:

- Porphyromonas gingivalis

- Prevotella intermedia

- Fusobacterium nucleatum

- Bacteroides (Tannerella) forsythensis and

- Treponema denticola.

Gram-positive bacteria have also been implicated since they can denude the available glycoproteins of their sugar chains, enabling the anaerobic Gram-negative proteolytic bacteria to break down the proteins. Gram negative bacteria can produce chemicals that produce malodour, which include in many instances

- volatile sulphur compounds (VSCs), mainly methyl mercaptan, hydrogen sulphide, and dimethyl sulphide

- diamines (putrescine and cadaverine) and

- short chain fatty acids (butyric, valeric and propionic). 
The evidence for the implication of other microorganisms, such as Helicobacter pylori, is scant.

The posterior area of the tongue dorsum is often the location of the microbial activity associated with bad breath. Debris, such as in patients with poor oral hygiene, or under a neglected or a poorly designed dental bridge or appliance is another cause. Any patient with oral cancer or a dry mouth can also develop oral malodour.

Defined infective processes that can cause malodour may include:

- Periodontal infections (especially necrotising gingivitis or periodontitis)

- Pericoronitis

- Other types of oral infections

- Infected extraction sockets

- Ulcers.

Improvement of oral hygiene, prevention or treatment of infective processes, and sometimes the use of antimicrobials can usually manage this type of oral malodour.

\section{Rare causes of oral malodour}

Systemic causes of oral malodour are rare but important and range from drugs to sepsis in the respiratory tract to metabolic disorders (Table 2).

\section{The complaint of oral malodour in the absence of malodour}

The complaint of oral malodour may be made by patients who do not have it but imagine it because of psychogenic reasons. This can be a real clinical dilemma, since no evidence of oral malodour can be detected even with objective testing, and the oral malodour may then be attributable to a form of delusion or monosymp-

\begin{tabular}{l} 
Table 2. Rare causes of oral malodour \\
\hline Drugs \\
Chloral hydrate \\
Cytotoxic drugs \\
Dimethyl sulphoxide \\
Nitrites and nitrates \\
Solvent abuse \\
Respiratory problems \\
Nasal sepsis \\
Tonsillitis \\
Sinusitis \\
Lower respiratory tract infection \\
Systemic disease \\
Gastrointestinal disease: (some believe in an \\
association with Helicobacter pylori infection) \\
Hepatic failure \\
Renal failure \\
Diabetic ketosis; the breath may smell of acetone. \\
Trimethylaminuria (fish-malodour syndrome); an \\
autosomal dominant metabolic disorder. \\
Trimethylamine (TMA) is produced by intestinal bacteria \\
on eating cholines (mainly in fish and eggs) and is \\
typically oxidised by a liver enzyme. Individuals with \\
trimethylaminuria lack this enzyme and thus secrete \\
TMA in various bodily fluids and via their breath. \\
Psychogenic factors \\
\end{tabular}

tomatic hypochondriasis (self-oral malodour; halitophobia).

Other people's behaviour, or perceived behaviour, such as apparently covering the nose or averting the face, is typically misinterpreted by these patients as an indication that their breath is indeed offensive. Such patients may have latent psychosomatic illness tendencies.

Many of these patients will adopt behaviour to minimise their perceived problem, such as

- covering the mouth when talking

- avoiding or keeping a distance from other people

- avoiding social situations

- using chewing gum, mints, mouthwashes or sprays designed to reduce malodour

- frequent toothbrushing

- cleaning their tongue.

Thus the oral hygiene may be superb in such patients. Medical help may be required to manage these patients.

Such patients unfortunately fail to recognise their own psychological condition, never doubt they have oral malodour and thus are often reluctant to visit a psychologic specialist.

\section{Summary}

Oral malodour can have a range of causes, though most cases of true malodour have an oral cause, and many others are imagined (Fig. 1).

\section{DIAGNOSIS OF ORAL MALODOUR}

Assessment of oral malodour is usually subjective by simply smelling exhaled air (organoleptic method) coming from the mouth and nose and comparing the two. Odour originating in the mouth, but not detectable from the nose is likely to be either oral or pharyngeal origin. Odour originating in the nose may come from the sinuses or nasal passages. Children sometimes place foreign bodies in the nose, leading to sepsis and malodour! Only in the rare cases in which similar odour is equally sensed coming from both the nose and mouth can one of the many systemic causes be inferred.

Specialist centres may have the apparatus for objectively measuring the responsible volatile sulphur compounds (methyl mercaptan, hydrogen sulphide, dimethyl sulphide) - a halimeter. Microbiological investigations such as the BANA (benzoyl-arginine-naphthylamide) test or darkfield microscopy can also be helpful.

\section{Management of oral malodour}

The management includes first determining which cases may have an extraoral aetiology.

A full oral examination is indicated and if an oral cause is likely or possible, management should include treatment of the cause, and other measures (see box).

In cases of malodour which may have an extraoral aetiology, the responsibility of the general dental practitioner is to refer the patient for 


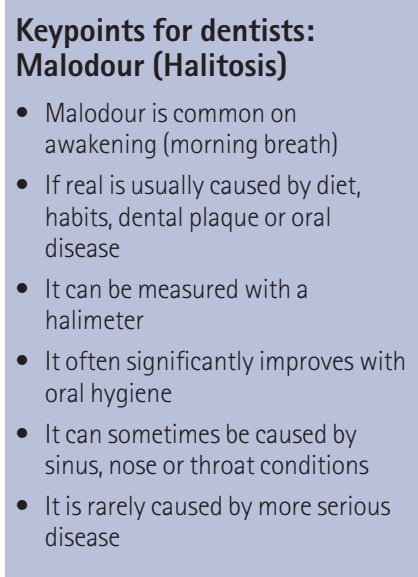

Key points for patients: 11 steps towards control of oral malodour

- Treat any identifiable cause (this may need antimicrobials)

- Avoid odiferous foods such as onions, garlic, spices and durian

- Avoid habits that may worsen breath odour, such as;

$$
\text { alcohol }
$$

tobacco

- Eat a good breakfast, and take regular meals including fresh fruit: an enzyme in pineapple (papain) helps clean the mouth

- Brush your teeth after meals

- Keep oral hygiene regular and good Prophylaxis

Toothbrushing

Flossing

- Rinse at least twice daily with chlorhexidine (eg Chlorohex, Corsodyl, Eludril), triclosan (Total), essential oils (Listerine), cetylpyridinium (MacLeans), chlorine dioxide (Retardex) or other mouthwashes

- Brush your tongue before going to bed: use a tongue scraper if that helps

- Keep your mouth as moist as possible by using

sugar-free chewing gums (eg Orbit, EnDeKay) diabetic sweets

- Use proprietary 'fresh breath' preparations eg Dentyl pH

- If you have dentures, leave them out at night and in hypochlorite (eg Dentural) or chlorhexidine.

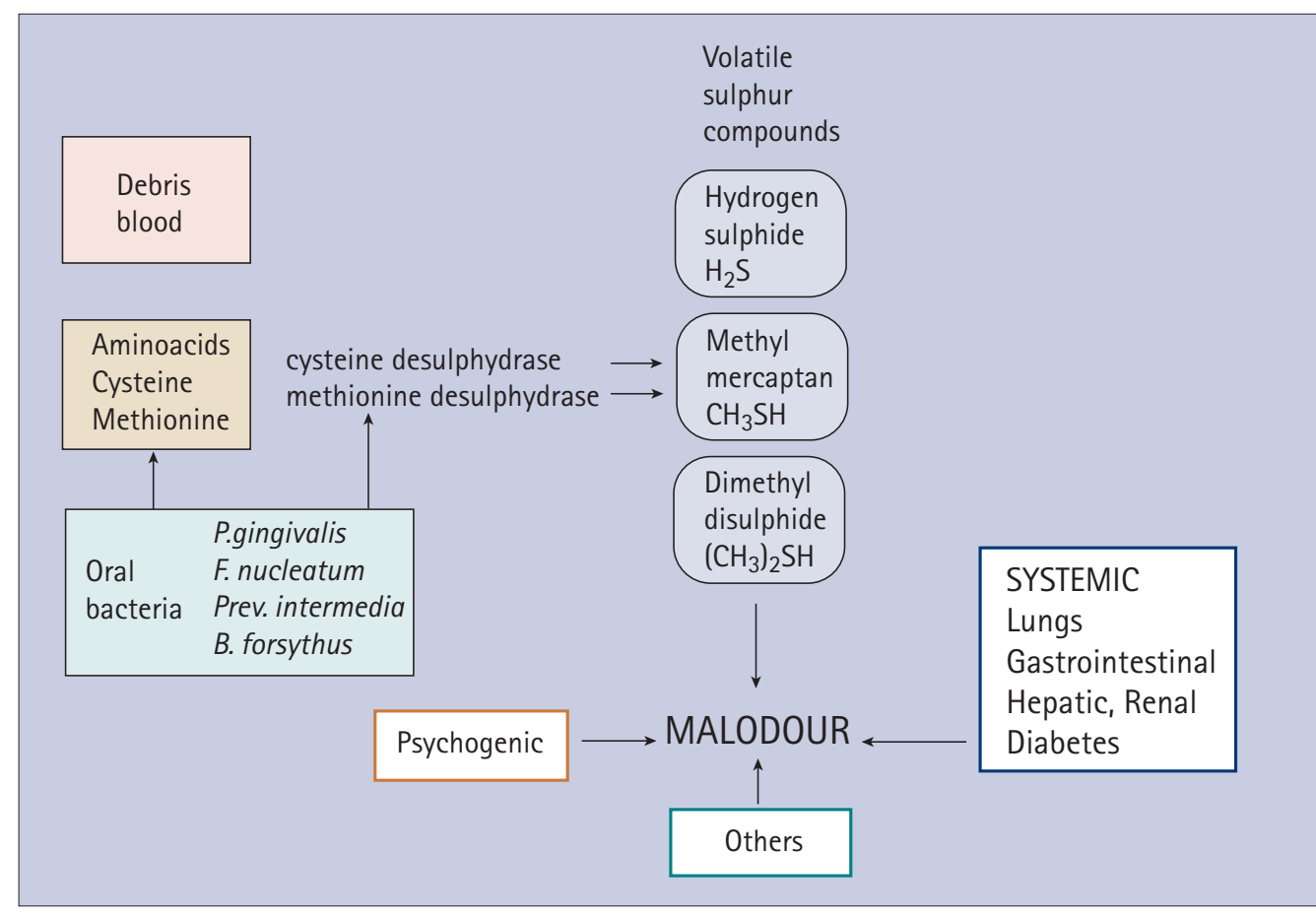

Fig. 1 Causes of malodour

evaluation to a specialist. This may involve an oral medicine opinion, an otorhinolaryngologist to rule out the presence of chronic tonsillitis or chronic sinusitis, a physician to rule out gastric, hepatic, endocrine, pulmonary, metabolic or renal disease or a psychologist or psychiatrist.

\section{Patient information and websites} http://www.tau.ac.il/ melros/

\section{Patients to refer}

Suspected systemic disease

Suspected malignancy 